\title{
Rotifera das zonas limnética e litorânea do reservatório de Tapacurá, Pernambuco, Brasil
}

\author{
Viviane L. dos S. Almeida ${ }^{1}$, Maria Eduarda L. de Larrazábal ${ }^{1}$, Ariadne do N. Moura ${ }^{2}$ \\ \& Mauro de Melo Júnior ${ }^{3}$
}

1. Departamento de Zoologia, Mestrado em Biologia Animal, Universidade Federal de Pernambuco, 50670-420 Recife, Pernambuco, Brasil.

2. Departamento de Biologia, Programa de Pós-Graduação em Botânica, Universidade Federal Rural de Pernambuco, 52171-900 Recife, Pernambuco, Brasil.

3. Departamento de Oceanografia, Mestrado em Oceanografia, Universidade Federal de Pernambuco.

\begin{abstract}
Rotifera from littoral and pelagic zones of Tapacurá reservoir, Pernambuco, Brazil. The rotifers of Tapacurá reservoir, State of Pernambuco, Brazil, were studied concerning the horizontal distribution in pelagic and littoral zones, in the rainy (August/2003) and dry seasons (January/2004). Qualitative and quantitative samples were carried out in nictimeral samplings in the different zones of the reservoir, with an interval of six hours in the two depths. Biotic parameters as richness, density, diversity and equitability were estimated. Similarity and Analysis of Variance (ANOVA) were also used. Twenty-eight species and two subspecies of Rotifer were found from which five species are considered new occurrences in State of Pernambuco. The littoral zone presented higher richness than the limnetic zone in both seasonal periods with nine exclusive species. The density and equitability differences between zones of the reservoir were not expressive in opposite to diversity that was higher in the littoral zone in the dry season ( $\mathrm{p}<0.01$ ). The similarity analysis indicated horizontal homogeneity of Rotifer community in the rainy season characterized by lower temperatures and higher wind velocity, and a tendency to horizontal stratification in the dry season characterized by higher temperatures and lower wind intensity. Presence of aquatic vegetation yielded peculiar features in the littoral zone.
\end{abstract}

KEYWORDS. Biotic limnology, horizontal distribution, Rotifer, biodiversity, aquatic vegetation.

RESUMO. Os rotíferos do reservatório de Tapacurá, Pernambuco, Brasil foram estudados quanto à distribuição horizontal nas zonas limnética e litorânea, nos períodos chuvoso (agosto de 2003) e seco (janeiro de 2004). Amostras quali-quantitativas foram obtidas através de coletas em ritmo nictimeral nas distintas zonas do reservatório, com intervalo de seis horas, em duas profundidades. Os parâmetros bióticos riqueza, densidade, diversidade e equitabilidade foram avaliados. Análises de similaridade e variância (ANOVA) também foram utilizadas. Vinte e oito espécies e duas subespécies de Rotifera foram encontradas, das quais cinco espécies são novas ocorrências para Pernambuco. A zona litorânea apresentou maior riqueza que a limnética em ambos ao período sazonais, com nove espécies exclusivas. As diferenças de densidade e equitabilidade entre as zonas do reservatório não foram significativas, ao contrário da diversidade, que apresentouse mais elevada na zona litorânea no período seco $(\mathrm{p}<0,01)$. A análise de similaridade revelou homogeneidade horizontal da comunidade de Rotifera no período chuvoso, caracterizado por menores temperaturas e maior velocidade do vento, e tendência à estratificação horizontal no período seco, caracterizado por temperaturas mais elevadas e menor intensidade dos ventos. A presença de macrófitas propiciou características peculiares na zona litorânea.

PALAVRAS-CHAVE. Limnologia biótica, distribuição horizontal, Rotifera, biodiversidade, macrófitas.

Reservatórios são lagos artificiais considerados ambientes de transição entre rios e lagos. O conhecimento dos organismos aquáticos e da cadeia alimentar de um reservatório é de grande importância, pois a presença ou ausência de certas espécies e a composição das comunidades existentes servem como indicadores do "status" da qualidade da água (STRAsKraba \& TundisI, 2000). De acordo com Pinto-Coelho (2004), o intenso programa governamental de construção de reservatórios vem demandando esforço considerável da comunidade científica na busca do entendimento sobre o funcionamento desses ecossistemas.

Horizontalmente, os lagos podem ser divididos em duas zonas: litorânea e limnética. A primeira está em contato direto com o ecossistema terrestre adjacente, sendo considerada um ecótono. A zona limnética ou pelágica corresponde à área que não sofre influência direta do ecossistema terrestre adjacente (EsTEVEs, 1998).

A zona litorânea geralmente apresenta grande quantidade de vegetação marginal (macrófitas aquáticas), o que resulta em elevada produtividade primária e variedade de nichos ecológicos, consequiência da abundância de habitats e de recursos alimentares. Muitos organismos que habitam essa zona possuem adaptações características (WeTZEL \& LiKENS, 1991; NogUeira et al., 2003). Na zona limnética, os organismos estão mais expostos às mudanças no regime de vazão da massa líquida, que determina a importância do fluxo longitudinal, e também aos maiores níveis de estratificação vertical causada pela ação da radiação e dos ventos nos movimentos e mistura da água (STRASKRABa \& TUNDISI, 2000).

Dentre os organismos mais estudados que habitam os reservatórios, os rotíferos geralmente sobressaem em riqueza e densidade. Esses organismos constituem um elo fundamental das cadeias alimentares em águas continentais, cobrindo o nicho ecológico dos pequenos filtradores. Os rotíferos são ainda importantes indicadores de condições ecológicas específicas, sendo bastante utilizados na determinação do estado trófico em corpos d'água (Nordi \& Watanabe, 1978; Margalef, 1983; Berzins \& Pejler, 1989; Branco et al., 2000). 
No Brasil, muitos trabalhos têm enfocado a comunidade de rotíferos em reservatórios, principalmente em relação à composição taxonômica e seu papel como bioindicadores, a exemplo dos trabalhos de NORDI \& Watanabe (1978), Matsumura-Tundisi (1999), GünTZEL \& Rocha (1998). Porém, pouco se sabe sobre os padrões de distribuição horizontal desses organismos na massa d'água. No estado de Pernambuco, os estudos limnológicos são ainda escassos. Alguns poucos trabalhos sobre Rotifera já foram desenvolvidos (Neumann-Leitão, 1981; Neumann-Leitão \& Nogueira, 1986; NeumANN-LeITÃo \& SouZA, 1987), porém, o estudo dessa comunidade em reservatórios é praticamente inexistente, consistindo apenas de trabalhos acadêmicos não-publicados. Desta forma, o tema consiste em uma importante lacuna científica para esse Estado.

De acordo com as características peculiares a cada zona do reservatório, a comunidade de Rotifera deve apresentar-se heterogênea entre as mesmas. Assim, este trabalho objetivou estudar a distribuição horizontal dos rotíferos do reservatório de Tapacurá nas zonas litorânea e limnética a partir dos dados de riqueza, densidade, diversidade e equitabilidade.

\section{MATERIAL E MÉTODOS}

O reservatório de Tapacurá está localizado no município de São Lourenço da Mata, Zona da Mata de Pernambuco (08 02'14"'S, 35 09'46”'W), sendo produto do represamento do rio Tapacurá, afluente do rio Capibaribe, principal bacia hidrográfica do estado. Utilizado para o abastecimento de água da Região Metropolitana do Recife e proteção contra enchentes, constitui-se no maior sistema produtor de água para a região (SANTOS \& CARNAVAL, 2002). De acordo com a Companhia Pernambucana de Recursos Hídricos, nos últimos anos esse reservatório tem apresentado características eutróficas, sendo a maior parte dos poluentes despejos líquidos urbanos.

Foram obtidas amostras através de coletas em ritmo nictimeral com intervalo de 6 horas, nos dias 18 e 19 de agosto de 2003 (período chuvoso), 21 e 22 de janeiro de 2004 (período seco). As coletas foram realizadas nos intervalos de 07:30/08:00 (matutino); 13:30/14:00 (vespertino); 19:30/20:00 (noturno) e 01:30/02:00 (madrugada).

As amostras foram obtidas nas regiões litorânea e limnética do reservatório, ressaltando-se para a primeira o acúmulo de macrófitas aquáticas. Além da superfície, a profundidade referente ao limite de zona eufótica - LZE também foi representada, a qual, segundo EsTEVEs (1998), corresponde a $1 \%$ da radiação da superfície. Para determinação do LZE, multiplicou-se o valor da transparência pelo fator 3 , considerando-se para a noite os valores obtidos durante o dia.

Para fins qualitativos, as amostras foram tomadas utilizando-se rede de plâncton de $64 \mu \mathrm{m}$ de abertura de malha em arrastos superficiais de 3 minutos. Para fins quantitativos, as amostras de superfície foram obtidas com balde graduado, utilizando-se no LZE garrafa do tipo Van Dorn com capacidade para 2 L. Em ambos os casos, $10 \mathrm{~L}$ de água foram filtrados em peneira de PVC com mesma abertura de malha da rede.
As amostras foram fixadas com formol a $4 \%$ neutralizado com tetraborato de sódio. Em campo também foram obtidos dados referentes aos seguintes parâmetros hidrológicos: transparência - disco de Secchi; oxigênio dissolvido - oxímetro digital; temperatura e condutividade elétrica - condutivímetro digital; turbidez - turbidímetro digital e $\mathrm{pH}$ - potenciômetro de bancada. Os parâmetros climatológicos referentes à precipitação total e velocidade do vento $\left(\mathrm{m} \mathrm{s}^{-1}\right)$ foram obtidos pela estação 82900 Recife do Instituto Nacional de Meteorologia.

Subamostras foram analisadas sob estereomicroscópio em placa milimetrada de $6 \mathrm{~cm}^{2}$. A confirmação das espécies foi feita em microscópio invertido, cuja identificação baseou-se, sobretudo, em Pennak (1978), Koste (1978), Neumann-Leitão \& Souza (1987) e NeUmann-Leitão et al. (1989).

A freqüência de ocorrência (FO) foi determinada segundo a fórmula da CETESB (1978), na qual os dados em forma de porcentagem foram assim classificados: > $70 \%$, muito freqüente; $70 \%$ a $40 \%$, freqüente; $40 \%$ a $10 \%$, pouco freqüente e $<10 \%$, esporádico. Para a densidade populacional em cada intervalo de coleta, considerou-se a média entre as profundidades, a fim de melhor adequar os dados aos objetivos deste artigo.

A densidade populacional foi utilizada para cálculo dos índices de Diversidade (H') e Equitabilidade (E) de Shannon, através do software Diversit versão 1.2, assim como para a análise de similaridade entre as zonas do reservatório, utilizando o método UPGMA (unweighted pair-group, aritmetic average) e o índice de Bray-Curtis, através do software NTSYS pc 2.1. Análise de variância do tipo ANOVA com critério único também foi realizada para avaliar a significância da variação nos parâmetros bióticos (riqueza, densidade, diversidade e equitabilidade) em relação à distribuição horizontal, utilizando-se para isso o pacote estatístico BioEstat 3.0 (AYREs et al., 2003).

As amostras coletadas encontram-se depositadas no Laboratório de Biologia da Conservação do Departamento de Zoologia, Centro de Ciências Biológicas da Universidade Federal de Pernambuco.

\section{RESULTADOS E DISCUSSÃO}

A precipitação total observada no período chuvoso foi de 194,8 mm, mostrando-se menor que aquela apresentada no período seco, que foi de $249,9 \mathrm{~mm}$, tendo este último mês sido considerado atípico para este parâmetro. Quanto aos ventos, estes apresentaram maior velocidade no período chuvoso, com valores médios de $3,5 \mathrm{~m} \cdot \mathrm{s}^{-1}$ e $2,1 \mathrm{~m} \cdot \mathrm{s}^{-1}$, respectivamente.

De uma forma geral, não foram detectadas diferenças expressivas quanto aos parâmetros hidrológicos entre as zonas do reservatório dentro de cada período sazonal, salvo a diferença entre os valores de OD e turbidez no período seco (Tab. I). Quanto ao oxigênio dissolvido em particular, os valores apresentados podem ser considerados baixos (média em torno de 3,0 mg. $\mathrm{L}^{-1}$ ), pois, segundo GARCÉs (1984), as comunidades aeróbias requerem águas com concentração maior que 4,0 mg. $\mathrm{L}^{-1}$. 
Foram encontradas 28 espécies e duas subespécies de Rotifera distribuídas em 10 famílias (Tab. II). Cinco espécies foram consideradas novas ocorrências para ecossistemas continentais do estado de Pernambuco: Lecane imbricata Carlin, 1939; L. clara (Bryce, 1892), Asplanchnopus hyalinus Harrin, 1913; Hexarthra fennica (Levander, 1892) e Conochilus unicornis Rousselet, 1892. Essas espécies ocorreram nas duas zonas do reservatório, com exceção de $L$. imbricata, observada apenas na zona litorânea.

As famílias Brachionidae e Lecanidae foram as mais representativas. Para a primeira, a maioria das espécies habitou as duas zonas do reservatório, sendo mais freqüentes e apresentando densidades superiores às da família Lecanidae; esta última apresentou maior riqueza na zona litorânea.

A família Brachionidae é considerada uma das mais importantes para o zooplâncton de águas continentais, cujas espécies geralmente têm hábito planctônico, enquanto que Lecanidae é litorânea, estando relacionada ao bentos e perifiton, principalmente em locais ricos em vegetação, ocorrendo no plâncton como migrantes ocasionais (Hutchinson, 1967; Neumann-Leitão \& NogUeira, 1986). No estudo de VIEIRA et al. (2000) sobre o zooplâncton do açude São José dos Cordeiros, Estado da Paraíba, a maior representação qualitativa também foi observada para a família Brachionidae, tendo as espécies de Lecanidae ocorrido principalmente após as chuvas.

Quanto à distribuição horizontal da riqueza total, todos os táxons encontrados ocorreram na zona litorânea, e dentre estes, 20 espécies e duas subespécies foram registradas também na zona limnética, totalizando nove espécies de ocorrência exclusiva para a zona litorânea. Esta diferença horizontal na riqueza foi significativa tanto para o período chuvoso $(\mathrm{p}=0,009)$ como para o período $\operatorname{seco}(\mathrm{p}=0,010)$.

Tabela I. Valores médios diários dos parâmetros hidrológicos nos períodos chuvoso (agosto 2003) e seco (janeiro 2004), durante o estudo da comunidade de Rotifera do reservatório de Tapacurá, Pernambuco, Brasil. *Os valores apresentados para a precipitação total e velocidade média do vento se referem aos dados diários para a estação meteorológica mais próxima.

\begin{tabular}{|c|c|c|c|c|}
\hline \multirow[t]{2}{*}{ PARÂMETROS } & \multicolumn{2}{|c|}{$\begin{array}{c}\text { PERÍODO } \\
\text { CHUVOSO } \\
\text { Zona do } \\
\text { reservatório }\end{array}$} & \multicolumn{2}{|c|}{$\begin{array}{c}\text { PERÍODO } \\
\text { SECO } \\
\text { Zona do } \\
\text { reservatório }\end{array}$} \\
\hline & Limnética & Litorânea & Limnética & Litorânea \\
\hline Transparência (m) & 0,85 & 0,85 & 0,85 & 0,40 \\
\hline $\begin{array}{l}\text { Limite de zona eufótica } \\
(\mathrm{m})\end{array}$ & 2,5 & 2,5 & 2,5 & 1,2 \\
\hline Temperatura $\left({ }^{\circ} \mathrm{C}\right)$ & 27,0 & 27,4 & 28,5 & 28,7 \\
\hline $\begin{array}{l}\text { Condutividade elétrica } \\
\left(\mu \mathrm{m} \cdot \mathrm{cm}^{-1}\right)\end{array}$ & 230,7 & 226,6 & 243,7 & 245,9 \\
\hline $\begin{array}{l}\text { Oxigênio dissolvido } \\
\left(\mathrm{mg} \cdot \mathrm{L}^{-1}\right)\end{array}$ & 3,6 & 3,5 & 2,5 & 3,6 \\
\hline $\mathrm{pH}$ & 7,9 & 7,9 & 7,6 & 7,8 \\
\hline Turbidez (NUT) & 16,0 & 17,1 & 24,2 & 12,8 \\
\hline $\begin{array}{l}\text { Precipitação total } \\
(\mathrm{mm})^{*}\end{array}$ & \multicolumn{2}{|c|}{194,8} & \multicolumn{2}{|c|}{249,9} \\
\hline $\begin{array}{l}\text { Velocidade média do } \\
\text { vento }\left(\mathrm{m} \cdot \mathrm{s}^{-1}\right)^{*}\end{array}$ & \multicolumn{2}{|c|}{3,5} & \multicolumn{2}{|c|}{2,1} \\
\hline
\end{tabular}

A maior riqueza verificada na zona litorânea foi provavelmente devido a sua localização na zona de ecótono e pela presença de vegetação flutuante abundante. De acordo com NogueIRA et al. (2003), ao se considerar a comunidade zooplanctônica, o número de espécies na zona limnética de um lago é geralmente menor que o verificado nas comunidades litorais. Isso se deve principalmente à heterogeneidade de habitats proporcionada por uma zona litorânea bem desenvolvida, com a presença de diferentes espécies de macrófitas. DAJOZ (1983) relata que nas regiões marginais dos lagos ocorre o chamado "efeito de borda" por ser uma zona de transição, apresentando uma fauna mais rica que nas biocenoses adjacentes, cujas espécies se misturam.

Deste modo, o enriquecimento de espécies na zona litorânea do reservatório de Tapacurá se deu pela presença tanto de espécies euplanctônicas - que estão adaptadas a habitar horizontalmente toda a coluna d'água - como de organismos do bentos, nêuston, e perifiton, adaptados para a vida nesse ecótono.

Na zona limnética também se observou grande quantidade de espécies não-planctônicas, cujos indivíduos podem ter sido trazidos do fundo por ação dos ventos, principalmente no período chuvoso, ou carreados a partir da margem, pela intensa precipitação observada durante o período seco. Porém, a influência das macrófitas aquáticas parece ter sido o fator determinante para explicar o fato. DABÉs (1995), estudando o zooplâncton na zona limnética de cinco lagoas marginais do rio São Francisco em Minas Gerais, encontrou predominância de espécies litorâneas e semiplanctônicas. StARLING (2000) também registrou predominância de espécies litorâneas na zona limnética da lagoa Bonita, no Distrito Federal, que se apresentou durante o estudo quase completamente coberta por macrófitas. PANARELLI et al. (2003) observaram predominância de espécies nãoplanctônicas na zona limnética nos trechos mais lóticos do sistema de lagoas da planície de inundação do rio Paraná, onde a zona litorânea tem maior influência.

Quanto à densidade total, no período chuvoso (Fig. 1), a zona limnética apresentou maior quantidade de organismos que a litorânea em quase todos os horários. No período seco (Fig. 2) se deu o inverso, tendo a litorânea sobressaído na densidade de organismos. Porém, essas diferenças não foram consideradas significativas ( $p>0,05$ em ambos os períodos sazonais), indicando que a densidade total da comunidade de Rotifera não foi diferente quanto à distribuição horizontal dentro de cada período sazonal.

Brachionus calyciflorus calyciflorus Pallas, 1766 e Keratella tropica (Apstein, 1907), da família Brachionidae, destacaram-se quanto à densidade populacional nas zonas litorânea e limnética em ambos os períodos sazonais (Tab. III). Enquanto B. calyciflorus calyciflorus apresentou maior densidade na zona limnética, $K$. tropica obteve maior ocorrência na zona litorânea. Essas espécies são bastante comuns em ecossistemas continentais brasileiros (REID \& TURNER, 1988; NeUmann-LeitÃo et al., 1989; ArCiFa et al., 1998; STARLING, 2000), podendo ocorrer em ambientes oligotróficos a eutróficos (Matsumura-Tundisi et al., 1990; STARLING, 2000). 
Tabela II. Distribuição horizontal, freqüência de ocorrência (FO) (MF, muito freqüente; F, freqüente; PF, pouco freqüente; E, esporádico) e hábito (PL, planctônico; NPL, não-planctônico) dos Rotifera do reservatório de Tapacurá, Pernambuco, Brasil.

\begin{tabular}{|c|c|c|c|c|}
\hline \multirow[t]{2}{*}{ ESPÉCIES } & \multicolumn{2}{|c|}{ DISTRIBUIÇÃO HORIZONTAL } & \multirow[t]{2}{*}{ FO } & \multirow[t]{2}{*}{ HÁBITO } \\
\hline & Zona limnética & Zona litorânea & & \\
\hline \multicolumn{5}{|l|}{ PHILODINIDAE } \\
\hline Rotaria sp. 1 & $\mathrm{x}$ & $\mathrm{x}$ & PF & NPL \\
\hline Rotaria sp. 2 & $\mathrm{x}$ & $\mathrm{x}$ & $\mathrm{PF}$ & NPL \\
\hline Rotaria sp. 3 & $\mathrm{x}$ & $\mathrm{x}$ & PF & NPL \\
\hline \multicolumn{5}{|l|}{ EPIPHANIDAE } \\
\hline Epiphanes macrouros (Barrois \& Daday, 1894) & $\mathrm{x}$ & $\mathrm{x}$ & $\mathrm{MF}$ & PL \\
\hline \multicolumn{5}{|l|}{ BRACHIONIDAE } \\
\hline Platyias quadricornis (Ehrenberg, 1832) & $\mathrm{x}$ & $\mathrm{x}$ & $\mathrm{E}$ & NPL \\
\hline Brachionus quadridentatus (Hermann, 1783) & - & $\mathrm{x}$ & $\mathrm{E}$ & NPL \\
\hline Brachionus calyciflorus calyciflorus Pallas, 1766 & $\mathrm{x}$ & $\mathrm{x}$ & $\mathrm{MF}$ & PL \\
\hline Brachionus calyciflorus anuraeiformis (Brehm, 1909) & $\mathrm{x}$ & $\mathrm{x}$ & $\mathrm{E}$ & PL \\
\hline Brachionus dolabratus Harring, 1915 & $\mathrm{x}$ & $\mathrm{x}$ & $\mathrm{F}$ & PL \\
\hline Brachionus falcatus Zacharias, 1898 & $\mathrm{x}$ & $\mathrm{x}$ & $\mathrm{F}$ & PL \\
\hline Brachionus havanaensis Rousselet, 1911 & $\mathrm{x}$ & $\mathrm{x}$ & $\mathrm{MF}$ & PL \\
\hline Keratella tropica (Apstein, 1907) & $\mathrm{x}$ & $\mathrm{x}$ & $\mathrm{MF}$ & PL \\
\hline Keratella americana Carlin, 1943 & $\mathrm{x}$ & $\mathrm{x}$ & MF & PL \\
\hline Anuraeopsis fissa (Gosse, 1851) & $\mathrm{x}$ & $\mathrm{x}$ & PF & PL \\
\hline \multicolumn{5}{|l|}{ COLURELLIDAE } \\
\hline Lepadella patella (O. F. Müller, 1786) & - & $\mathrm{x}$ & $\mathrm{PF}$ & NPL \\
\hline \multicolumn{5}{|l|}{ LECANIDAE } \\
\hline Lecane imbricata Carlin, 1939 & - & $\mathrm{x}$ & $\mathrm{E}$ & NPL \\
\hline Lecane clara (Bryce, 1892) & $\mathrm{x}$ & $\mathrm{x}$ & PF & NPL \\
\hline Lecane ludwigi (Eckstein, 1883) & $\mathrm{x}$ & $\mathrm{x}$ & PF & NPL \\
\hline Lecane curvicornis (Murray, 1913) & - & $\mathrm{x}$ & $\mathrm{PF}$ & PL \\
\hline Lecane papuana (Murray, 1913) & - & $\mathrm{x}$ & $\mathrm{E}$ & NPL \\
\hline Lecane (Monostyla) lunaris (Ehrenberg, 1832) & $\mathrm{x}$ & $\mathrm{x}$ & $\mathrm{E}$ & NPL \\
\hline Lecane (Monostyla) bulla (Gosse, 1886) & $\mathrm{x}$ & $\mathrm{x}$ & $\mathrm{F}$ & $\mathrm{PL} / \mathrm{NPL}$ \\
\hline Lecane sp. & - & $\mathrm{x}$ & PF & NPL \\
\hline \multicolumn{5}{|l|}{ NOTOMATIDAE } \\
\hline Cephalodella sp. 1 & - & $\mathrm{x}$ & $\mathrm{E}$ & NPL \\
\hline Cephalodella sp. 2 & - & $\mathrm{x}$ & PF & NPL \\
\hline \multicolumn{5}{|l|}{ TRICHOCERCIDAE } \\
\hline Trichocerca sp. & - & $\mathrm{x}$ & $\mathrm{E}$ & $\mathrm{PL} / \mathrm{NPL}$ \\
\hline \multicolumn{5}{|l|}{ SYNCHAETIDAE } \\
\hline Ploesoma truncatum (Levander, 1894) & $\mathrm{x}$ & $\mathrm{x}$ & $\mathrm{E}$ & PL/NPL \\
\hline \multicolumn{5}{|l|}{ ASPLANCHINIDAE } \\
\hline Asplanchnopus hyalinus Harring, 1913 & $\mathrm{x}$ & $\mathrm{X}$ & $\mathrm{F}$ & PL \\
\hline \multicolumn{5}{|l|}{ HEXARTHRIDAE } \\
\hline Conochilus unicornis Russelet, 1892 & $\mathrm{x}$ & $\mathrm{x}$ & $\mathrm{F}$ & $?$ \\
\hline Hexarthra fennica (Levander, 1892) & $\mathrm{x}$ & $\mathrm{x}$ & $\mathrm{F}$ & $?$ \\
\hline
\end{tabular}

Em relação à diversidade (Figs. 3, 4), a zona litorânea obteve valores mais elevados que a limnética, tanto no período chuvoso quanto no período seco, variando entre valores baixos e médios (1,46 a 2,66 bit.ind $\left.{ }^{-1}\right)$, sendo esta diferença, porém, significativa apenas para o período seco $(p=0,009)$. De acordo com Pinto-CoelHo (2002), habitats muito variados e heterogêneos favoreceriam o aumento da diversidade por oferecerem mais combinações de micro-habitats e nichos ecológicos, o que pode explicar a maior diversidade observada para a zona litorânea no presente estudo. CRispim \& WatANABE (2000) encontram maior diversidade na zona limnética nos açudes São José dos Cordeiros e Taperoá, no semi-árido paraibano, ao contrário do ocorrido no reservatório de Tapacurá, o que pode estar relacionado à ausência ou reduzida presença de macrófitas aquáticas naqueles ambientes.

Os valores de equitabilidade foram, de forma geral, superiores a 0,5 , mostrando boa distribuição dos indivíduos entre as diferentes espécies. Porém, enquanto no período chuvoso a zona limnética obteve maiores valores em relação à litorânea (Fig. 5), no período seco ocorreu o contrário, tendo a litorânea sobressaído quanto 
a este parâmetro (Fig. 6); essas diferenças não foram significativas ( $p>0,05$ em ambos os períodos sazonais).

$\mathrm{Na}$ análise de similaridade entre as zonas do reservatório para cada período sazonal, a correlação entre a matriz de similaridade e a matriz cofenética foi alta $(0,99)$, revelando que os dados estão bem ajustados, tendo o dendrograma (Fig. 7) apresentado três níveis de agrupamento. No primeiro nível (grupo A) a correlação foi de 0,55 , a qual, de acordo com o coeficiente utilizado (Bray-Curtis), indica pouca similaridade entre os macrocomponentes do grupo, separando desta forma os períodos sazonais. Os dois níveis seguintes agruparam as zonas do reservatório em cada período sazonal, tendo o grupo B (período seco) obtido correlação de 0,28 e o grupo $\mathrm{C}$ (período chuvoso) correlação de 0,07 , indicando maior similaridade entre as zonas do reservatório no período chuvoso em relação ao período seco.
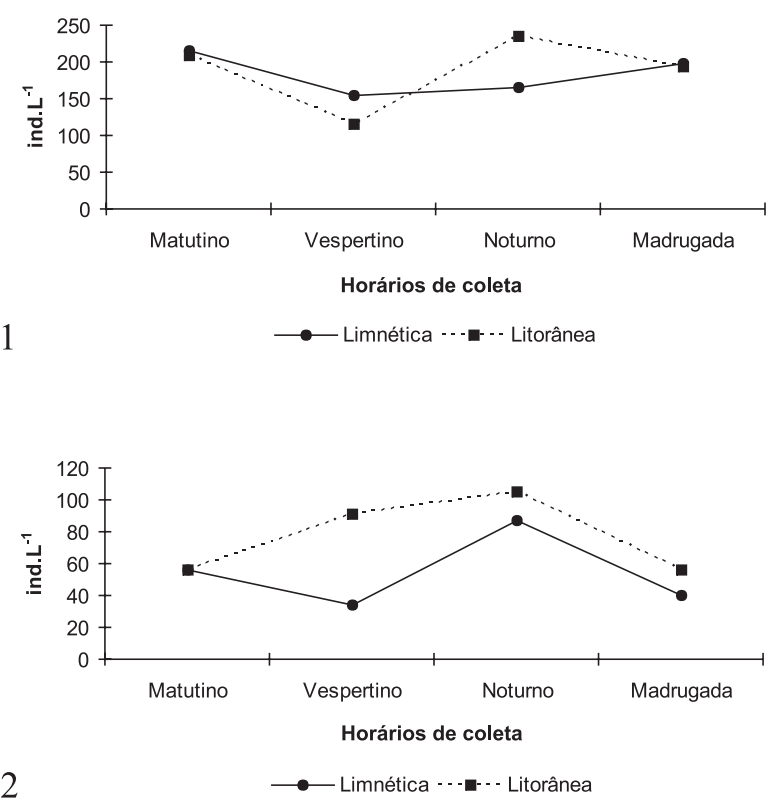

Figs. 1, 2. Variação diária da densidade total (ind. $\left.\mathrm{L}^{-1}\right)$ da comunidade de Rotifera nas zonas litorânea e limnética do reservatório de Tapacurá, Pernambuco, Brasil (1, período chuvoso; 2, período seco).

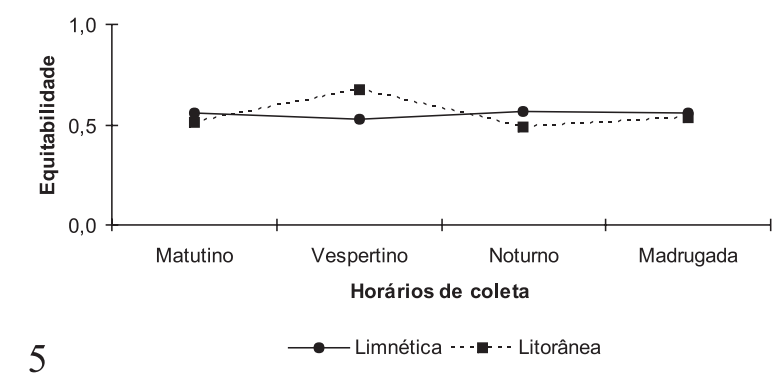

Os dados de similaridade apresentados revelam a influência da variação sazonal na distribuição horizontal da comunidade de rotíferos no ambiente estudado. Assim, no período chuvoso, em que as temperaturas são menores e a velocidade do vento é maior, observou-se maior homogeneidade horizontal, com diferença significativa apenas para a riqueza específica $(\mathrm{p}=0,009)$, que foi maior na zona litorânea. Já no período seco, caracterizado por temperaturas mais elevadas, menor intensidade dos ventos e maiores diferenças nos níveis de turbidez entre as zonas do reservatório, detectou-se maior heterogeneidade horizontal da comunidade, o qual apresentou diferenças significativas na riqueza específica $(\mathrm{p}=0,010)$ e na diversidade $(\mathrm{p}=0,009)$, com valores mais elevados também na zona litorânea, revelando tendência à estratificação horizontal da comunidade de rotíferos neste período.
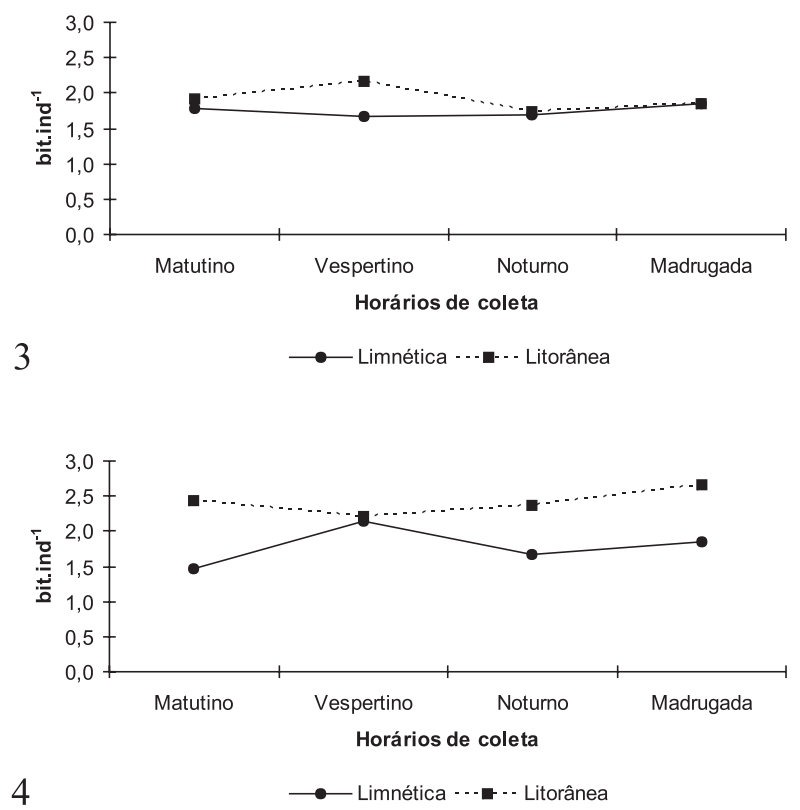

Figs. 3, 4. Variação diária da diversidade (bit.ind ${ }^{-1}$ ) da comunidade de Rotifera nas zonas litorânea e limnética do reservatório de Tapacurá, Pernambuco, Brasil (3, período chuvoso; 4, período seco)

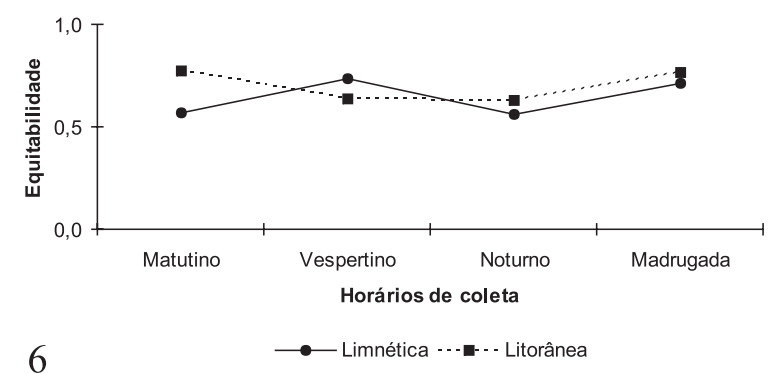

Figs. 5, 6. Variação diária da equitabilidade da comunidade de Rotifera nas zonas litorânea e limnética do reservatório de Tapacurá, Pernambuco, Brasil: 5, período chuvoso; 6 , período seco. 

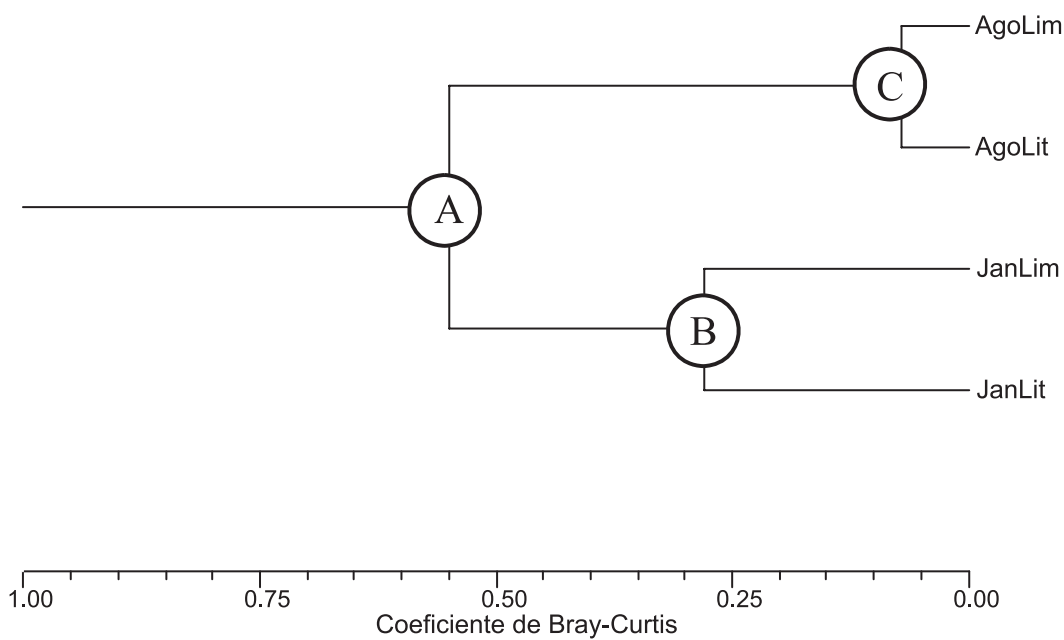

Fig. 7. Dendrograma da análise de similaridade dos dados de densidade populacional diária da comunidade de Rotifera, nos períodos chuvoso (Ago) e seco (Jan) entre as zonas litorânea (Lit) e limnética (Lim) do reservatório de Tapacurá, Pernambuco, Brasil.

Tabela III. Densidade populacional média da comunidade de Rotifera do reservatório de Tapacurá, Pernambuco, Brasil, em agosto 2003 (período chuvoso) e janeiro 2004 (período seco).

\begin{tabular}{|c|c|c|c|c|c|c|c|c|}
\hline \multirow{3}{*}{ Espécies } & \multicolumn{4}{|c|}{ Período chuvoso } & \multicolumn{4}{|c|}{ Período Seco } \\
\hline & \multicolumn{2}{|c|}{ Limnética } & \multicolumn{2}{|c|}{ Litorânea } & \multicolumn{2}{|c|}{ Limnética } & \multicolumn{2}{|c|}{ Litorânea } \\
\hline & ind. $\mathrm{L}^{-1}$ & $\%$ & ind. $\mathrm{L}^{-1}$ & $\%$ & ind. $L^{-1}$ & $\%$ & ind. $\mathrm{L}^{-1}$ & $\%$ \\
\hline Rotaria sp. 1 & - & - & 0,13 & 0,07 & - & - & 0,81 & 1,05 \\
\hline Rotaria sp. 2 & - & - & 0,13 & 0,07 & 0,06 & 0,12 & 0,06 & 0,08 \\
\hline Rotaria sp. 3 & 0,06 & 0,03 & 0,25 & 0,13 & - & - & 0,75 & 0,97 \\
\hline Epiphanes macrouros & 6,80 & 3,70 & 5,50 & 2,93 & 3,50 & 6,45 & 4,50 & 5,83 \\
\hline Platyias quadricornis & - & - & 0,06 & 0,03 & - & - & - & - \\
\hline Brachionus quadridentatus & - & - & 0,06 & 0,03 & - & - & - & - \\
\hline Brachionus calyc. calyciflorus & 52,16 & 28,36 & 45,06 & 24,02 & 26,50 & 48,85 & 19,94 & 25,81 \\
\hline Brachionus dolabratus & 3,28 & 1,78 & 2,75 & 1,47 & - & - & 0,06 & 0,08 \\
\hline Brachionus falcatus & 2,71 & 1,47 & 2,06 & 1,10 & 0,38 & 0,69 & 0,63 & 0,81 \\
\hline Brachionus havanaensis & 1,83 & 0,99 & 2,63 & 1,40 & 2,94 & 5,41 & 2,88 & 3,72 \\
\hline Keratella tropica & 103,38 & 56,19 & 110,56 & 58,93 & 19,13 & 35,25 & 30,88 & 39,97 \\
\hline Keratella americana & 8,33 & 4,53 & 7,19 & 3,83 & 0,63 & 1,15 & 0,38 & 0,49 \\
\hline Anuraeopsis fissa & - & - & - & - & 0,25 & 0,46 & 0,31 & 0,40 \\
\hline Lepadella patella & - & - & 0,13 & 0,07 & - & - & 0,38 & 0,49 \\
\hline Lecane imbricata & - & - & - & - & - & - & 0,13 & 0,16 \\
\hline Lecane clara & - & - & - & - & 0,13 & 0,23 & 0,50 & 0,65 \\
\hline Lecane ludwigi & 0,69 & 0,37 & 0,38 & 0,20 & - & - & 0,13 & 0,16 \\
\hline Lecane curvicornis & - & - & - & - & - & - & 0,25 & 0,32 \\
\hline Lecane papuana & - & - & - & - & - & - & 0,06 & 0,08 \\
\hline Lecane (Monostyla) lunaris & - & - & - & - & 0,06 & 0,12 & 0,06 & 0,08 \\
\hline Lecane (Monostyla) bulla & 0,19 & 0,10 & 2,50 & 1,33 & 0,06 & 0,12 & 1,75 & 2,27 \\
\hline Lecane sp. & - & - & 0,25 & 0,13 & - & - & - & - \\
\hline Cephalodella sp. 1 & - & - & 0,13 & 0,07 & - & - & - & - \\
\hline Cephalodella sp. 2 & - & - & 1,06 & 0,57 & - & - & - & - \\
\hline Ploesoma truncatum & - & - & 0,13 & 0,07 & - & - & - & - \\
\hline Asplanchnopus hyalinus & 0,13 & 0,07 & - & - & 0,44 & 0,81 & 6,31 & 8,17 \\
\hline Conochilus unicornis & 0,25 & 0,14 & 0,19 & 0,10 & 0,06 & 0,12 & 6,19 & 8,01 \\
\hline Hexarthra fenica & 4,18 & 2,27 & 6,50 & 3,46 & 0,13 & 0,23 & 0,31 & 0,40 \\
\hline Total & 183,96 & 100,00 & 187,63 & 100,00 & 54,25 & 100,00 & 77,25 & 100,00 \\
\hline
\end{tabular}


Agradecimentos. A Giulliari de Lira, Antônio Travassos e Ênio Wocyli, alunos do Programa de Pós-Graduação em Botânica da UFRPE, pelo apoio em campo e laboratório. Ao Dr. Manoel Flores e à M.Sc. Eliane Cavalcanti pela concessão de material para coleta. A Paulo Martins, coordenador da Reserva Ecológica do Tapacurá, pelo apoio logístico, assim como ao barqueiro Isael dos Santos e demais funcionários da reserva. À Dra. Sigrid Neumann Leitão, da UFPE, pela ajuda na confirmação das espécies e tratamento dos dados. Ao CNPq pela concessão da bolsa de mestrado à primeira autora. E, finalmente, os autores expressam seus agradecimentos aos revisores anônimos, pelas sugestões e críticas valiosas.

\section{REFERÊNCIAS BIBLIOGRÁFICAS}

Arcifa, M. S.; Silva, L. H. S. DA \& Silva, M. H. L. DA. 1998. The planktonic community in a tropical brazilian reservoir: composition, fluctuations and interactions. Revista Brasileira de Biologia 58(2):241-254.

Ayres, M.; Ayres, M., Jr.; Ayres, D. L. \& Santos, A. S. 2003. BioEstat 3.0: aplicações estatísticas nas áreas das ciências biológicas e médicas. Belém, Sociedade Civil Mamirauá. 290p.

Berzins, B. \& Pejler, B. 1989. Rotifer occurrence in relation to oxygen content. Hydrobiologia 183:165-172.

Branco, C. W.; Kolowsky-Suzuky, B.; Esteves, F. A. \& Coimbra e SouzA, L. 2000. Rotifers as ecological indicators in three coastal lagoons in the state of Rio de Janeiro. In: Simpósio DE Ecossistemas Brasileiros, 5º, Vitória. Anais... Vitória, UFES. p. 249-261.

CETESB (Companhia de Tecnologia de Saneamento Ambiental). 1978. Determinação do zooplâncton marinho: métodos qualitativos e quantitativos. Normatização técnica L5301. São Paulo, Cetesb. 12p.

Crispim, M. C. \& Watanabe, T. 2000. Heterogeneidade no ecossistema lacustre, baseado na comunidade zooplanctônica de açudes. In: Simpósio de Ecossistemas Brasileiros, $5^{\circ}$, Vitória. Anais... Vitória, UFES. p.431-441.

DABÉs, M. B. G. S. 1995. Composição e descrição do zooplâncton de 5 (cinco) lagoas marginais do rio São Francisco, Pirapora/ Três Marias/Minas Gerais, Brasil. Revista Brasileira de Biologia 55(4):831-845.

Dajoz, R. 1983. Ecologia geral. Petrópolis, Vozes. 472p.

Esteves, F. DE A. 1998. Fundamentos de Limnologia. 2ed. Rio de Janeiro, Interciência. 602p.

GARCÉs, B. H. A. 1984. Estudio preliminar sobre el zooplancton del lago Bayano, Panamá. Revista de Biologia Tropical 32(1):17-24.

Güntzel, A. \& RochA, O. 1998. Relações entre a comunidade zooplanctônica e as condições tróficas da lagoa Caconde, Osório, RS, Brasil. Iheringia, Série Zoologia, (84):56-71.

Hutchinson, G. E. 1967. A treatise on limnology. II. Introduction to lake biology and their limnoplankton. New York, John Wiley \& Sons. 1115p.

Koste, W. 1978. Rotatoria: Die Rädertiere Mitteleroupas Ein Bestimmungswerk begründet von Max Voigt. Uberordnung Monogonta. 2. Berlin, Gebrüder Borntraeger. 637p.

Margalef, R. 1983. Limnología. Barcelona, Omega. 1010p.

Matsumura-Tundisi, T. 1999. Diversidade de zooplâncton em represas do Brasil. In: Henry, R. ed. Ecologia de reservatórios: estrutura, função e aspectos sociais. Botucatu, FUNDIBIO/FAPESP. p.39-54.
Matsumura-Tundisi, T.; Neumann-Leitão, S.; Aguena, L. S. \& Miyahara, J. 1990. Eutrofização da Represa de Barra Bonita: estrutura e organização da comunidade de Rotifera. Revista Brasileira de Biologia 50(4):923-935.

Neumann-Leitão, S. 1981. Rotíferos de Pernambuco. I. Algumas espécies que ocorrem em viveiros de cultivo de camarão do Cabo - PE. In: Encontro de Zoologia do Nordeste, $3^{\circ}$, Recife. Anais... Recife. p.191-199.

Neumann-Leitão, S. \& Nogueira, J. D. DA C. 1986. Rotíferos, cladóceros e copépodos de Pernambuco. I. Algumas espécies que ocorrem em viveiros de cultivo de camarões de Nova Cruz. In: Encontro de Zoologia do Nordeste, $5^{\circ} / 6^{\circ}$, Teresina. Anais... Teresina. p. 87-118.

Neumann-Leitão, S. \& Souza, F. B. V. A. de. 1987. Rotíferos (Rotatoria) planctônicos do Açude de Apipucos, RecifePernambuco (Brasil). Arquivos de Biologia e Tecnologia 30(3):393-418.

Neumann-Leitão, S.; Nogueira-Paranhos, J. D. \& Souza, F. B. V. A DE. 1989. Zooplâncton do Açude de Apipucos, Recife-PE (Brasil). Arquivos de Biologia e Tecnologia 32(4):803-821.

Nogueira, M. G.; George, D. G. \& Jorcin, A. 2003. Estudo do zooplâncton em zonas litorâneas lacustres: um enfoque metodológico. In: Henry, R. ed. Ecótonos nas interfaces dos ecossistemas aquáticos. São Carlos, RiMa. p.83-126.

Nordi, N. \& WATANABE, T. 1978. Nota preliminar sobre os rotíferos (zooplâncton) do Açude Epitácio Pessoa, Boqueirão, Paraíba. Revista Nordestina de Biologia 1(1):31-39.

Panarelli, E.; Casanova, S. M. C.; Nogueira, M. G.; Mitsuka, P. M. \& Henry, R. 2003. A comunidade zooplanctônica ao longo de gradientes longitudinais no rio Paranapanema/represa de Jurumim (São Paulo, Brasil). In: Henry, R. ed. Ecótonos nas interfaces dos ecossistemas aquáticos. São Carlos, RiMa. p. $129-160$.

PENNAK, R. W. 1978. Fresh water invertebrates of the United States. New York, Wiley Interscience. 803p.

Pinto-Coelho, R. M. 2002. Fundamentos em ecologia. Porto Alegre, Artmed. 252p.

2004. Métodos de coleta, preservação, contagem e determinação de biomassa em zooplâncton de águas epicontinentais. In: Bicudo, C. E. DE M. \& Bicudo, D. DE C. eds. Amostragem em limnologia. São Carlos, RiMa. p.149-166.

Reid, J. W. \& Turner, P. N. 1988. Planktonic Rotifera, Copepoda and Cladocera from Lagos Açu and Viana, State of Maranhão, Brazil. Revista Brasileira de Biologia 48(3):485-495.

Santos, E. M. dos \& Carnaval, A. C. O. DE Q. 2002. Anfíbios Anuros do Estado de Pernambuco. In: Tabarelli, M. \& Silva, J. M. C. DA. eds. Diagnóstico da Biodiversidade de Pernambuco. Recife, SECTMA/Massangana. p. 529-535.

Starling, F. L. do R. M. 2000. Comparative study of the zooplankton composition of six lacustrine ecosystems in Central Brazil during the dry season. Revista Brasileira de Biologia 60(1):101-111.

Straskraba, M. \& Tundisi, J. G. 2000. Gerenciamento da qualidade da água de represas. In: Tundisi, J. G. ed. Diretrizes para o gerenciamento de lagos. São Carlos, ILEC/IEE. v.9, 280p.

Vieira, D. M.; Crispim, M. C. \& Watanabe, T. 2000. Impacto da cheia e da seca sobre a comunidade zooplanctônica do açude São José dos Cordeiros no semi-árido paraibano. In: SIMPósıo DE Ecossistemas Brasileiros, 5º, Vitória. Anais... Vitória, UFES p.401-407.

Wetzel, R. G. \& Likens, G. E. 1991. Limnological analysis. 2ed. New York, Springer-Verlag. 491p. 